\title{
Urea-Water-Solution Properties: Density, Viscosity, and Surface Tension in an Under-Saturated Solution
}

\author{
Sauli Halonen ${ }^{1}$, Teija Kangas ${ }^{2}$, Mauri Haataja ${ }^{1}$, Ulla Lassi ${ }^{3}$ \\ ${ }^{I}$ Department of Mechanical Engineering, P.O. Box 3000, FI-90014 University of Oulu, Finland. \\ ${ }^{2}$ University of Oulu, Research Unit of Sustainable Chemistry, P.O. Box 3000, FI-90014 University of Oulu, Finland. \\ ${ }^{3}$ University of Jyvaskyla, Kokkola University Consortium Chydenius, Unit of Applied Chemistry, Talonpojankatu 2B, \\ FI-67100 Kokkola, Finland.
}

Sauli Halonen, saulihal@student.oulu.fi

This is a post-peer-review, pre-copyedit version of an article published in Emiss. Control Sci. Technol. The final authenticated version is available online at: https://doi.org/10.1007/s40825-016-0051-1.

\begin{abstract}
A temperature-concentration dependent surface fit for the relative viscosity of a urea-water-solution (UWS) is calculated based on experimental and literature data. For the surface fit, a 2D Lorenzian function was used, where the xaxis was assigned to a urea mass fraction and the $y$-axis to the solution temperature and the rest of the parameters were optimized based on the experimental and literature data. The surface model describes the relative viscosity of undersaturated urea-water-solution (UWS). The experimental data for the kinematic viscosity was measured with an Ubbelohde capillary viscometer whose temperature was controlled with a thermostat. The temperature and concentration range was from 293.15 to $353.15 \mathrm{~K}$ in $10 \mathrm{~K}$ increments and for urea mass fractions from 0.325 to 0.7 . The kinematic viscosity values from the experiment were converted to relative viscosity by calculating the density of the UWS. An exponential fit was calculated to describe the specific gravity of the UWS based on literature data.

Additionally, the surface tension of the UWS was measured at room temperature $(293.15 \mathrm{~K})$ in a mass fraction range from 0.302 to 0.596 . As a result, simple models describing UWS properties were obtained and these models can be implemented into computational fluid dynamics (CFD) simulations.
\end{abstract}

Keywords: Urea-water-solution, viscosity, density, surface tension, measurement

Acknowledgments This work was made possible by Proventia Emission Control Oy located in Oulunsalo, Finland. We would like to thank Mr. Joni Kosamo from Oulu University of Applied Sciences for making the viscosity measurements possible.

\section{Nomenclature}

$\begin{array}{ll}\text { Symbol } & \text { Description } \\ A & \text { Surface fit parameter } \\ D & \text { Capillary diameter } \\ E & \text { Kinetic energy correction factor } \\ k & \text { Capillary constant } \\ L & \text { Capillary length } \\ T & \text { Temperature } \\ t & \text { Flow time } \\ V & \text { Effective flux volume } \\ w_{1} & \text { Surface fit parameter } \\ w_{2} & \text { Surface fit parameter }\end{array}$




$\begin{array}{ll}y_{c} & \text { Surface fit parameter } \\ x_{c} & \text { Surface fit parameter } \\ z_{0} & \text { Surface fit parameter }\end{array}$

\section{Greek symbols}

$\begin{array}{ll}\text { Symbol } & \text { Description } \\ \gamma & \text { Mass fraction } \\ v & \text { Viscosity } \\ \rho & \text { Density } \\ \sigma & \text { Surface tension }\end{array}$

\section{Subscripts}

Symbol

Description

water

Variable describes water

uws

Urea-water-solution

rel

Relative value 


\section{Introduction}

Selective catalytic reduction (SCR) is used in modern on- and off-road vehicles to reduce nitrogen oxide emissions (NOx) [1]. The method uses ammonia $\left(\mathrm{NH}_{3}\right)$ as a reducing agent for the chemical reactions. Instead of using ammonia in its gas phase, it is introduced to the system in the form of a urea-water-solution (UWS) through an injection nozzle. When the UWS is injected into the exhaust pipe, the small droplets heat up and start to evaporate and as the water content in the droplets decreases, the urea starts a thermal decomposition into $\mathrm{NH}_{3}$ and isocyanic acid (HNCO).

After the initial atomization at the nozzle, the droplets are carried by the exhaust gas flow. Depending on the flow conditions, the droplets will evaporate as a result of heat transfer from the surrounding gas or hit the walls of the system forming a fluid film which evaporates. The interaction between the wall and the droplets depends on multiple factors. The factors, which affect the outcome of the droplet impact are: the impact velocity, the direction of the velocity vector related to the surface, droplet size, properties of the droplet liquid, the surface interfacial tension, the roughness and wettability of the solid surface, as well as nonisothermal effects such as solidification and evaporation, and air entrapment [2]. In this work we will discuss the properties of UWS used in SCR systems, particularly the viscosity, density and surface tension of the UWS.

Different models for droplet-wall-interaction take advantage of dimensionless variables such as the Weber number, the droplet Reynolds number, the Ohnesorge number, and their derivatives [2]. In these variables, viscosity, density, and surface tension are factors alongside the droplet diameter and velocity. For SCR applications the UWS wall interactions are critical because deposits can be formed under certain conditions [3]. The droplets hit the walls of the exhaust system at different stages of the evaporation. During the evaporation the concentration of the droplets changes, thus changing the material properties of the solution and affecting the behavior at the wall.

The viscosities of UWS have been reported in [4-9]. Chadwell [4] measured the UWS viscosity at 278.19, 283.13, $288.19,293.16$, and $298.22 \mathrm{~K}$ temperatures with $0.05,0.10$, and 0.15 urea mass fractions, and from a urea mass fraction of 0.0168 and 0.195 at the temperature of $283.13 \mathrm{~K}$. Whereas Kawahara et al. [5] measured the viscosity of UWS at $298.15 \mathrm{~K}$ for a urea mass fraction ranging from 0.0569 to 0.603 . Toryanik [6] reported measuring the viscosity between 278.15 and $343.15 \mathrm{~K}$ and on a range up to 0.270 urea mass fraction but the article lacks detailed tabulated data and only presents results in a graph. Jones [7] reported results for dilute solutions of UWS at room temperature. The measured urea mass fraction range was from $0.12 \mathrm{E}-4$ to 0.0112 .

The official data sheet by chemical company BASF [9] gives a dynamic viscosity value of $1.4 \mathrm{mPas}$ at $298.15 \mathrm{~K}$ which corresponds to a kinematic viscosity of $1.29 \mathrm{~mm}^{2} / \mathrm{s}$ when the density of UWS is calculated according to [5]. Jäger et el. [8] did viscosity and density measurements on UWS at a temperature range from 293.15 to $363.15 \mathrm{~K}$, and on a urea mass fraction range from 0.20 to 0.80 . The density of UWS has also been measured by Chadwell [4], Perman [10], and Jones [7]. Chadwell measured the densities of UWS solutions in the same range as the viscosity. In Perman's study, the temperature range for the density measurement was from 313.17 to $353.25 \mathrm{~K}$ and for the urea mass fraction, from 0.072 to 0.81 while Jones [7] measured the density of UWS at $298.15 \mathrm{~K}$ in mass fractions between 0.12E-4 and 11.99E-3.

The surface tension of UWS reported by Birkhold et al. [11] was $75 \mathrm{mN} / \mathrm{m}$ at 303.15 K. Whereas UWS supplier BASF [9] gives a value of $65 \mathrm{mN} / \mathrm{m}$ at $293.15 \mathrm{~K}$. According to the best knowledge of the authors these are the only values found in the literature. Since this information is not easily acquirable, the surface tension, in CFD simulations, is usually simplified to a constant value or values of pure water have been used. For example, Birkhold et al. [11] neglected the difference between UWS and water in lower concentrations, and Ström et al. [12] used values for water instead of UWS in their simulation.

As discussed above, the material properties of UWS are not well presented in the application range used in SCR systems. In this study the data is collected from the literature and experiments, and presented in a way which is usable for computational fluid dynamics (CFD) simulations. The aim is to determine the properties of UWS in temperature and concentration range used in SCR solutions. The models can be then used to get better approximations on the dropletwall interactions in SCR systems. 


\section{Experimental}

In this study the viscosity of UWS was measured from 293.15 to $353.15 \mathrm{~K}$ in $10 \mathrm{~K}$ increments and for urea mass fractions from 0.325 to 0.7 . The described range contains a supersaturated area which was excluded from this study. In the surface tension measurement, the temperature was kept constant at room temperature $(293.15 \mathrm{~K})$ and mass fractions from 0.325 to 0.596 were measured. The density values for UWS used in this study were obtained from the literature.

\subsection{Materials}

The samples for the viscosity and surface tension measurement were prepared by adding pure urea into commercially available standard UWS (AdBlue). One solution with a lower concentration than standard was prepared by adding deionized water to the standard UWS. The standard UWS is manufactured according to the ISO 22241-1 standard where the tolerance for the urea mass fraction is $0.325 \pm 0.007$. The purity of the additional urea used was higher than $98.5 \%$ and it was acquired from Sigma-Aldrich.

\subsection{Measurement of kinematic viscosity}

The viscosity measurement was done in the laboratory of the Oulu University of Applied Sciences. An Ubbelohde capillary viscometer was used with a LAUDA iVisc measurement device. The device resolution was 0.01 seconds and the viscosity range minimum was $0.30 \mathrm{~mm}^{2} / \mathrm{s}$. Two different capillary types were used: I and $0 \mathrm{c}$ with capillary constants of 0.01 and 0.003 , respectively. The device was calibrated with specific calibration fluid before the measurements were taken. For the temperature management the LAUDA Viscotemp $15 \mathrm{~S}$ was used. The temperature stability of the device is $\pm 0.01 \mathrm{~K}$.

Each sample was measured six times of which the first two were premeasurements and the four latter the main measurements. The aim of the premeasurements was to make sure the sample had reached the desired temperature before the actual measurement. An average flow time was calculated from the four main measurements and the kinematic viscosity was calculated. The standard deviation limit was set to 0.5 seconds for the temperatures below $333.15 \mathrm{~K}$ and it was increased to 0.8 seconds at the higher temperatures. This was done because the viscosity approached the minimum measurable value with the device and thus the deviation was affected.

The software automatically defines the kinematic viscosity with Equation 1.

$$
v=k \cdot(t-\Delta t)
$$

where $v$ is kinematic viscosity, $k$ is the capillary constant, $t$ is the flow time in seconds, and $\Delta t$ is the kinetic energy correction time. The kinetic energy correction is used to compensate for the pressure loss at the inlet and outlet ends of the capillary. The Hagenbach kinetic energy correction time is defined according to,

$$
\Delta t=\frac{E}{k \cdot t^{2}}
$$

where $E$ is the kinetic energy correction factor [13]. The kinematic energy correction factor is defined according to:

$$
E=\frac{0.0166 V^{3 / 2}}{L(D k)^{1 / 2}}
$$

where $V$ is effective flux volume, $L$ is length of the capillary, and $D$ is the capillary diameter [14]. 
The measured urea mass fractions were $0.325,0.373,0.426,0.471,0.5,0.584$ and 0.7 . Fig. 1 presents the measurement points in relation to the liquidus line. Below this line the urea starts precipitating into the solution. The urea-water phase diagram is presented similarly to Babkina et al. [15] and it is based on literature data from [16-19].

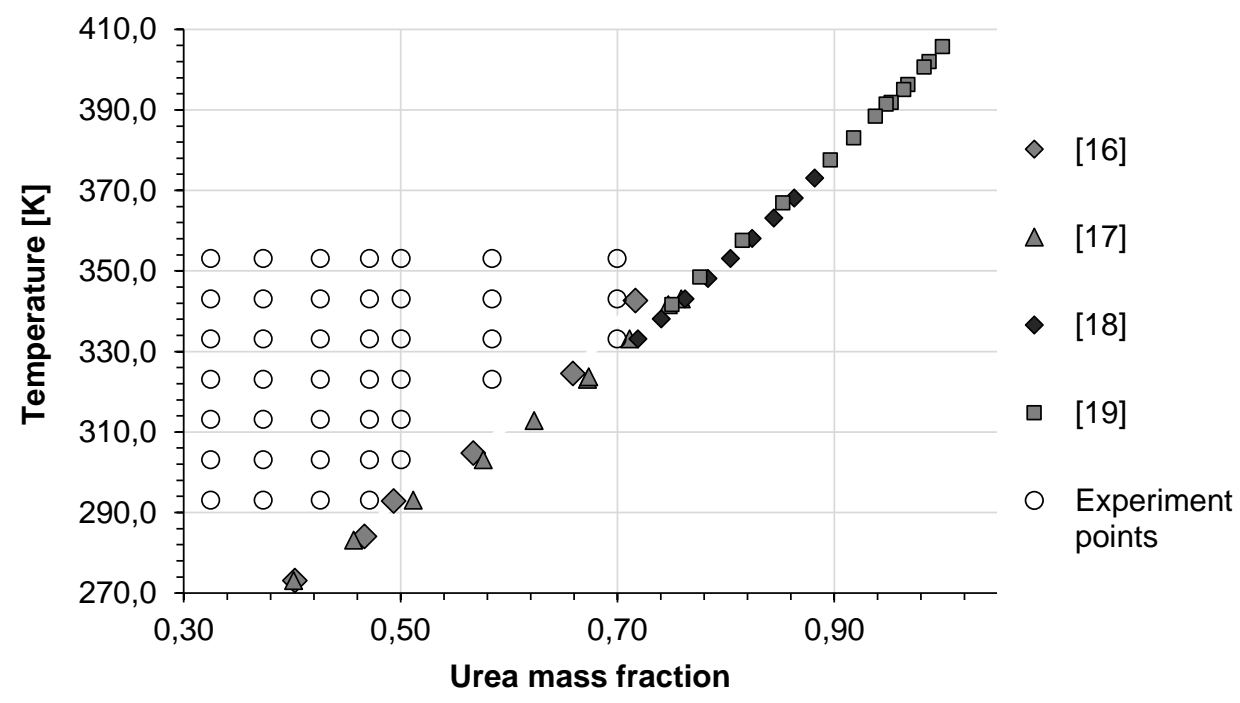

Fig. 1 Measurement points in the experiment are presented as open circles. The liquidus line of UWS for a fully saturated solution, obtained from the literature, is presented with filled symbols [16-19]

\subsection{Measurement of surface tension}

The surface tension measurement was performed in the laboratory of the Research unit of Sustainable Chemistry, at The University of Oulu. A manual device with the Du Noüy ring method was used to determine the surface tension of the solution. The device resolution was $0.5 \mathrm{mN} / \mathrm{m}$. The measurement device did not have the ability to use a thermostat but the temperature of the sample was kept at room temperature with $\pm 0.3 \mathrm{~K}$ accuracy.

The measurement device was calibrated with deionized water at room temperature at the beginning of each measurement set and a correction factor was calculated. The water reference values were compared with values from the IAPWS [20]. The largest deviation from the IAPWS values was $0.38 \mathrm{mN} / \mathrm{m}$ but the average deviation was 0.16 $\mathrm{mN} / \mathrm{m}$ with five different calibrations. The maximum standard deviation from the calibrations was $0.27 \mathrm{mN} / \mathrm{m}$.

Urea mass fractions of $0.302,0.325,0.373,0.426$, and $0.471,0.501$, and 0.596 were measured. The total number of individual measurement from each concentration sample was 10. During the measurements with the highest mass fraction, urea started to precipitate to the surface of the measurement ring. This might decrease the concentration of the solution slightly and have an effect on the surface shape at the measurement ring, thus having an effect on the results.

\subsection{Calculation of density}

To make the comparison easier between the viscosity measurement results and the results from the literature, all the values were converted to relative viscosity. For this conversion the density of water and the density of UWS was needed. For the water density the equation presented by Kell [21] was used, 


$$
\begin{gathered}
\rho_{\text {water }}(T)=\left(999.83952+16.945176 T-7.9870401 \times 10^{-3} T^{2}\right. \\
-46.170461 \times 10^{-6} T^{3}+105.56302 \times 10^{-9} T^{4} \\
\left.-280.54253 \times 10^{-12} T^{5}\right) /\left(1+16.879850 \times 10^{-3} T\right)
\end{gathered}
$$

where $\mathrm{T}$ is the temperature of water in degrees Celsius. (The viscosity of water was calculated based on the IAPWS model [22]). For the density of the UWS an exponential function was defined to fit the literature data $[4,7,8,10]$. From the density values reported in the literature, the specific gravity of the UWS was calculated by using the density of water from Equation 4 as a reference. The equation for the exponential fit of specific gravity is,

$$
\frac{\rho_{u w s}}{\rho_{\text {water }}(T)}=0.9999 e^{0.255 \gamma}
$$

where $\rho_{u w s}$ is the density of UWS and $\gamma$ is the urea mass fraction. The $\mathrm{R}^{2}$ value for the exponential fit is 0.9989 . The calculated specific gravity values and the exponential fit is shown in Fig. 2.

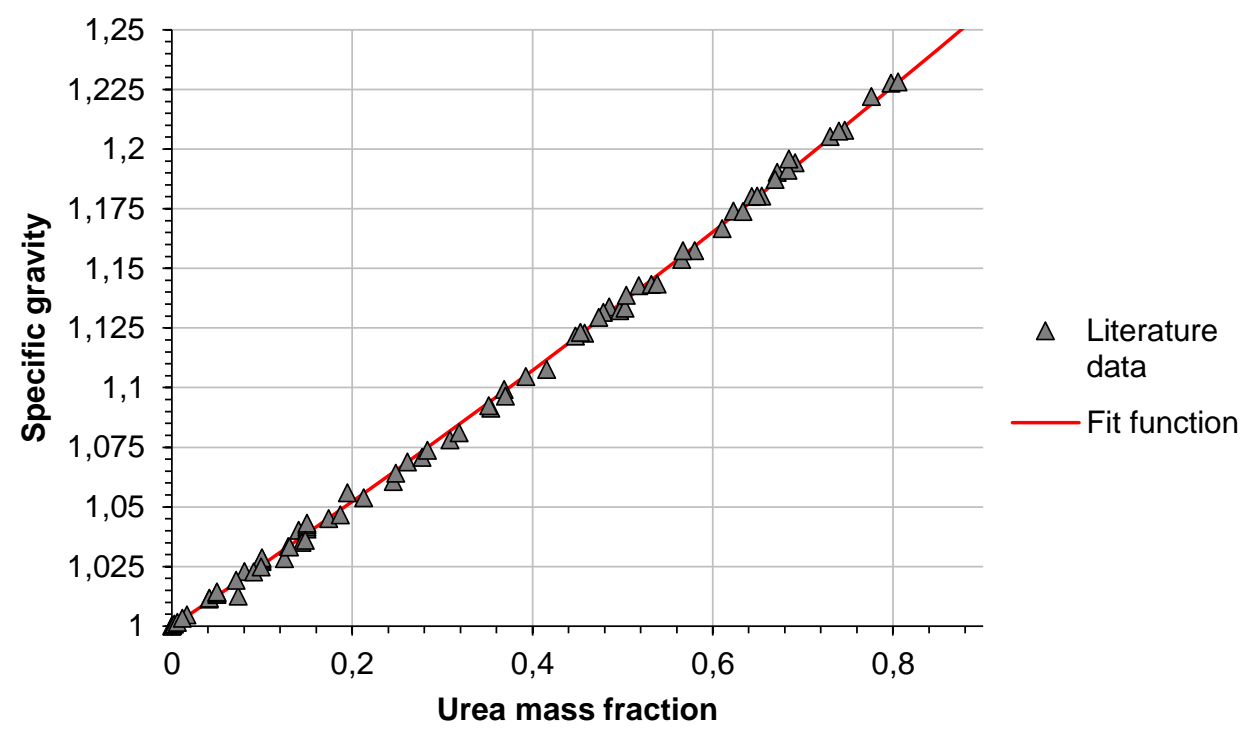

Fig. 2 The specific gravity of UWS with different urea mass fractions. The data is obtained from the literature $[4,7,8,10]$ and an exponential function is fit to the values, shown in the solid line

The values given by Equation 5 were compared to the literature data and the maximum deviation was $1.164 \%$ with the data from Jones [15]. The average deviations with the other reported values are presented in Table 1.

\begin{tabular}{ccccc}
\hline $\begin{array}{c}\text { Average } \\
\text { deviation [\%] }\end{array}$ & Jones [7] & Jäger et al. [8] & Perman [10] & Chadwell [4] \\
\hline
\end{tabular}

Table 1 The average deviation of the UWS density model with measured values from the literature [7, 8, 10, 4]

The model gives the density of UWS in a temperature range from 278 to $364 \mathrm{~K}$ and in a urea mass fraction range from 0 to 0.8 . The accuracy of Equation 5 cannot be confirmed completely in this area due to the lack of literature data. The mass fractions from 0.0 to 0.1 in a temperature range from 298 to $363 \mathrm{~K}$ lacks data as does the area where the urea mass fraction is from 0.2 to 0.4 in a temperature range from 270 to $290 \mathrm{~K}$. In the oversaturated region, the accuracy of Equation 5 cannot be confirmed. Only Perman [10] has measured densities of slightly oversaturated solutions but these 
measurement points were close to the liquidus line so Equation 5 still gives reasonable density values. The measured density points from the literature are presented in Fig. 3 .

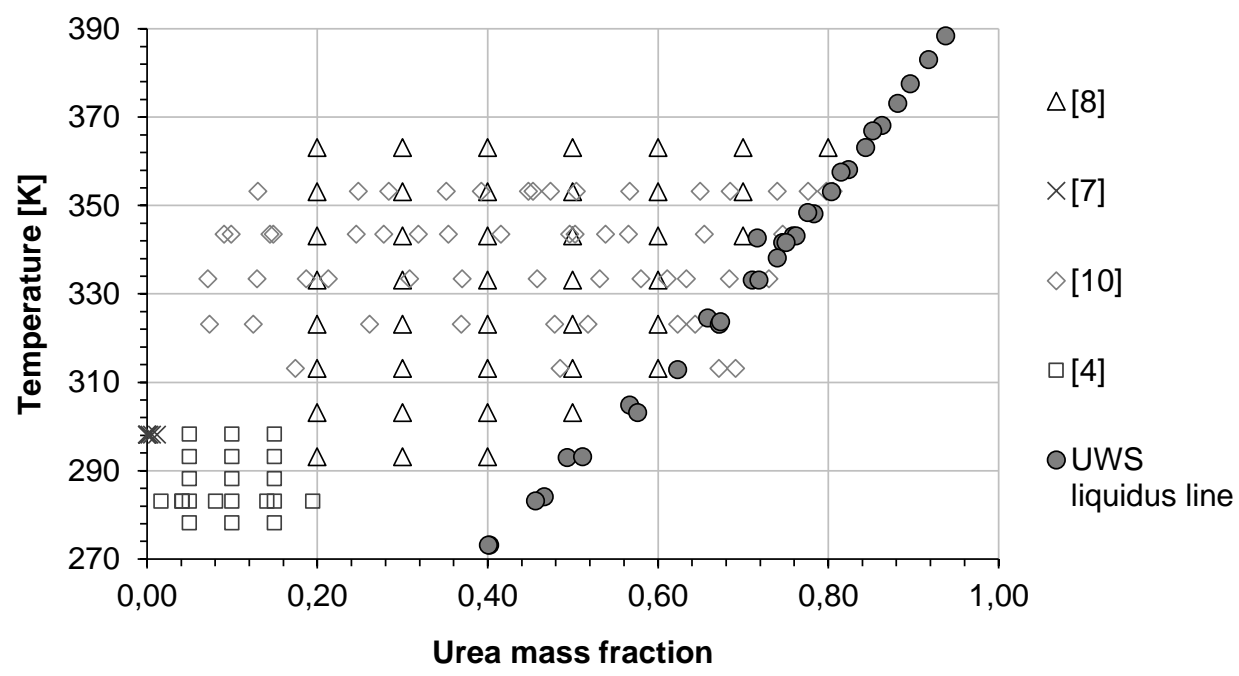

Fig. 3 Location of different density measurements found in the literature $[4,7,8,10]$. The liquidus line is shown with filled circles 


\section{Results}

\subsection{Viscosities}

The results from the viscosity measurement are show in Table 2. Temperature is given in both degrees Kelvin and Celsius. The urea mass fraction gives the urea mass relation to the solution mass e.g. 100 grams of UWS contains 32.5 grams of urea. In the right-hand column the relative viscosity of the solution is presented.

\begin{tabular}{|c|c|c|c|c|c|}
\hline $\begin{array}{c}\text { Temperature } \\
{[\mathrm{K}]}\end{array}$ & $\begin{array}{c}\text { Temperature } \\
{\left[{ }^{\circ} \mathbf{C}\right]}\end{array}$ & $\begin{array}{l}\text { Urea mass } \\
\text { fraction }\end{array}$ & $\begin{array}{c}\text { Kinematic } \\
\text { viscosity }\left[\mathrm{mm}^{2} / \mathrm{s}\right]\end{array}$ & $\begin{array}{c}\text { Calculated density of } \\
\text { UWS }\left[\mathrm{kg} / \mathrm{m}^{3}\right]\end{array}$ & $\begin{array}{c}\text { Relative } \\
\text { viscosity* }\end{array}$ \\
\hline \multirow{3}{*}{293.15} & \multirow{3}{*}{20} & 0.325 & 1.2592 & 1084.35 & 1.366 \\
\hline & & 0.373 & 1.3310 & 1097.76 & 1.462 \\
\hline & & 0.426 & 1.4621 & 1112.61 & 1.627 \\
\hline \multirow{5}{*}{303.15} & \multirow{5}{*}{30} & 0.325 & 1.0127 & 1081.57 & 1.377 \\
\hline & & 0.373 & 1.0886 & 1094.94 & 1.498 \\
\hline & & 0.426 & 1.1806 & 1109.76 & 1.647 \\
\hline & & 0.471 & 1.3065 & 1122.71 & 1.844 \\
\hline & & 0.500 & 1.3578 & 1131.01 & 1.930 \\
\hline \multirow{5}{*}{313.15} & \multirow{5}{*}{40} & 0.325 & 0.8570 & 1077.84 & 1.418 \\
\hline & & 0.373 & 0.9280 & 1091.17 & 1.555 \\
\hline & & 0.426 & 0.9918 & 1105.93 & 1.684 \\
\hline & & 0.471 & 1.0829 & 1118.84 & 1.860 \\
\hline & & 0.500 & 1.1318 & 1127.12 & 1.958 \\
\hline \multirow{6}{*}{323.15} & \multirow{6}{*}{50} & 0.325 & 0.7317 & 1073.30 & 1.440 \\
\hline & & 0.373 & 0.7799 & 1086.57 & 1.554 \\
\hline & & 0.426 & 0.8477 & 1101.28 & 1.712 \\
\hline & & 0.471 & 0.9188 & 1114.13 & 1.877 \\
\hline & & 0.500 & 0.9902 & 1122.37 & 2.038 \\
\hline & & 0.584 & 1.1508 & 1146.67 & 2.419 \\
\hline \multirow{7}{*}{333.15} & \multirow{7}{*}{60} & 0.325 & 0.6322 & 1068.05 & 1.452 \\
\hline & & 0.373 & 0.6773 & 1081.25 & 1.575 \\
\hline & & 0.426 & 0.7330 & 1095.88 & 1.727 \\
\hline & & 0.471 & 0.7932 & 1108.67 & 1.891 \\
\hline & & 0.500 & 0.8324 & 1116.87 & 1.999 \\
\hline & & 0.584 & 0.9985 & 1141.05 & 2.450 \\
\hline & & 0.700 & 1.3763 & 1175.16 & 3.477 \\
\hline \multirow{7}{*}{343.15} & \multirow{7}{*}{70} & 0.325 & 0.5572 & 1062.15 & 1.469 \\
\hline & & 0.373 & 0.5928 & 1075.28 & 1.583 \\
\hline & & 0.426 & 0.6433 & 1089.83 & 1.741 \\
\hline & & 0.471 & 0.6925 & 1102.55 & 1.896 \\
\hline & & 0.500 & 0.7297 & 1110.71 & 2.012 \\
\hline & & 0.584 & 0.8742 & 1134.75 & 2.463 \\
\hline & & 0.700 & 1.1847 & 1168.67 & 3.438 \\
\hline \multirow{7}{*}{353.15} & \multirow{7}{*}{80} & 0.325 & 0.4960 & 1055.66 & 1.482 \\
\hline & & 0.373 & 0.5280 & 1068.72 & 1.597 \\
\hline & & 0.426 & 0.5720 & 1083.18 & 1.754 \\
\hline & & 0.471 & 0.6172 & 1095.82 & 1.914 \\
\hline & & 0.500 & 0.6487 & 1103.92 & 2.027 \\
\hline & & 0.584 & 0.7688 & 1127.82 & 2.454 \\
\hline & & 0.700 & 1.0591 & 1161.53 & 3.481 \\
\hline
\end{tabular}

Table 2 Tabulated values from the viscosity measurements. * The relative viscosity is calculated by using the densities from Equations 4 and 5 
The kinematic viscosity values given in Table 2. are plotted in Fig. 4. The results are consistent at different temperatures but a urea mass fraction of 0.471 deviates from the curvature at 303.15 and $313.15 \mathrm{~K}$. At $323.15 \mathrm{~K}$. The urea mass fraction of 0.50 appears to have a slightly higher value in reference to the overall curvature. Other than the mentioned values, the results seem to be in line with each other.

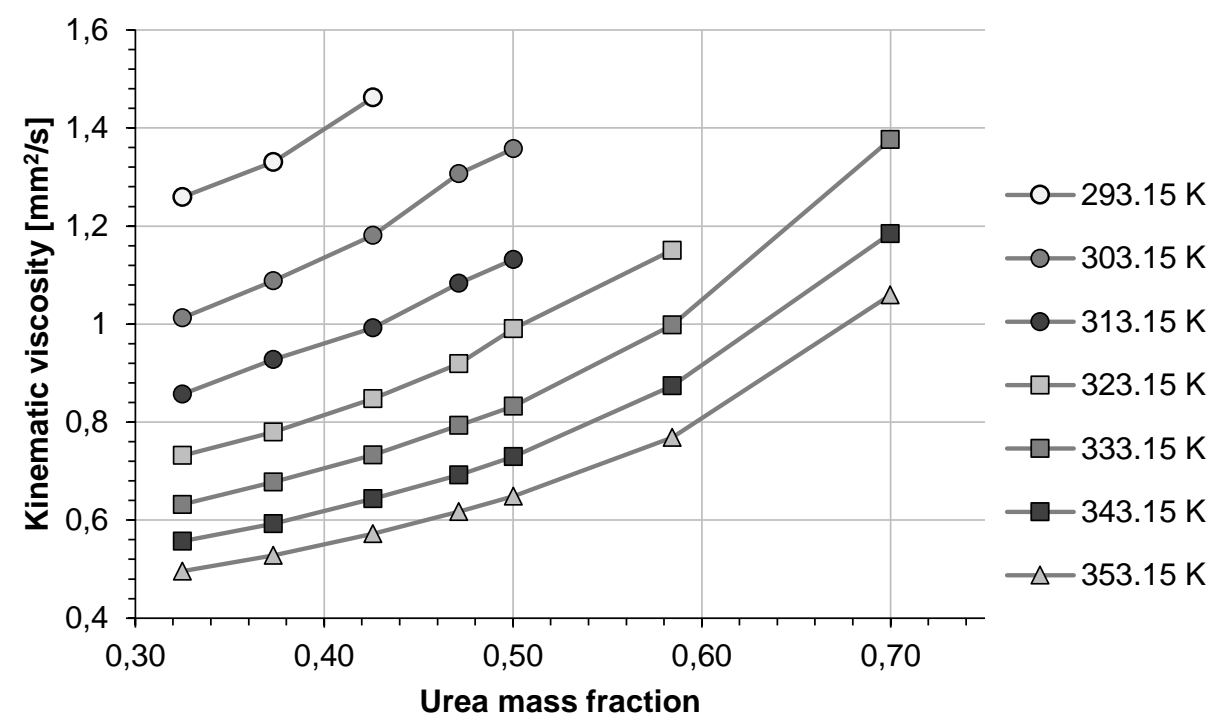

Fig. 4 The results from viscosity measurements in a temperature range from 293.15 to $352.15 \mathrm{~K}$ with different urea mass fractions

\subsection{Surface tension}

The results of the surface tension measurement for the UWS are shown in Table 3. and in Fig. 5. The results presented here were calculated as an average over the measurement series. The large deviation in the results is thought to be due to manual measurement and the large resolution of the device. The deviation and the resolution problems were countered by increasing the number of samples and measurements, but the deviation still remained rather high. Other affecting factors were the temperature control of the sample and the possibility of dynamic surface tension effects.

\begin{tabular}{cc}
$\begin{array}{c}\text { Surface } \\
\text { tension } \\
{[\mathbf{m N} / \mathbf{m}]}\end{array}$ & $\begin{array}{c}\text { Urea mass } \\
\text { fraction }\end{array}$ \\
\hline 74.03 & 0.302 \\
74.76 & 0.325 \\
74.68 & 0.373 \\
75.07 & 0.426 \\
75.56 & 0.471 \\
76.26 & 0.501 \\
77.46 & 0.596 \\
\hline
\end{tabular}

Table 3 Tabulated values from the surface tension measurements 


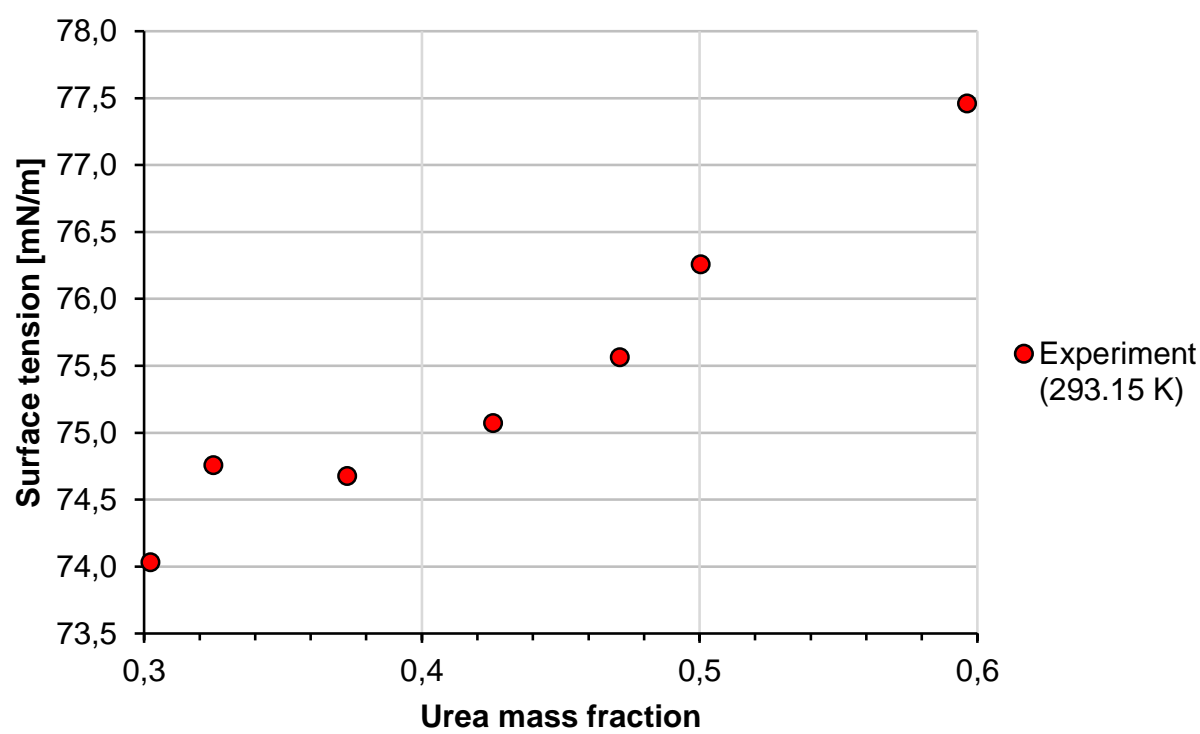

Fig. 5 Surface tension of UWS at room temperature with varying urea mass fractions 


\section{Discussion}

\subsection{Viscosity}

Chadwell [4] measured the viscosity of UWS at low temperatures and concentrations. The experimental results cannot be compared directly to these values since they are apart in the temperature - mass fraction plane. When combined with the results from Jäger et al. [8] the gap is filled. Fig. 6 shows the comparison of our measurements with Chadwell [4], Jäger et al. [8], Kawahara et al. [5], and Jones [7] with different urea mass fractions. The values from the literature and measurements seems to fit together well in the big picture. At higher urea mass fractions our results deviated slightly from the values measured by Jäger et al. [8]. The difference between the results occur for both temperatures, $343.15 \mathrm{~K}$ and $353.15 \mathrm{~K}$. This might be caused by the difference in the solution concentration between the experiments.

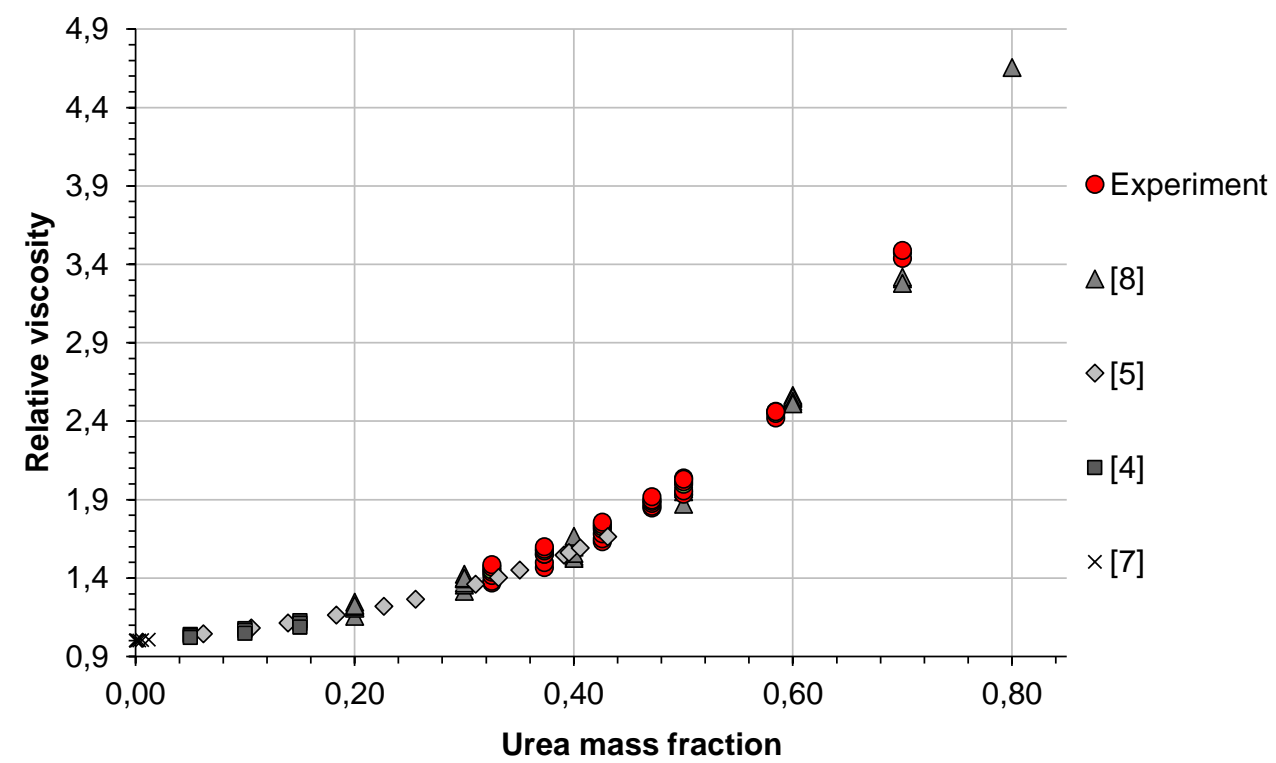

Fig. 6 Our measurements in relation with the values found in the literature $[4,5,7,8]$

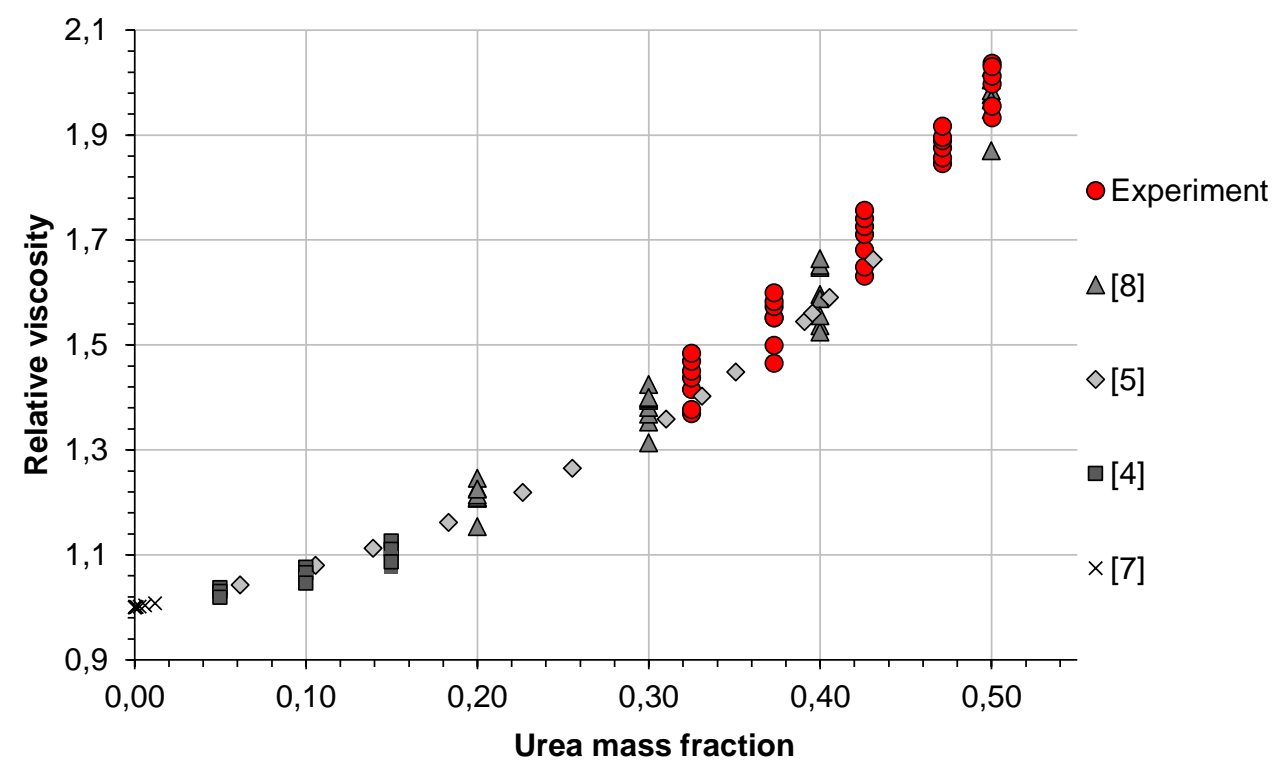

Fig. 7 Magnification on the lower mass fraction areas of the result comparison [4, 5, 7, 8]. 
The relative viscosity values given in Table 2. are compared with the literature data in Fig. 6 and a magnification of the low concentration area is presented in Fig. 7. When the urea mass fraction approaches zero, the relative viscosity approaches unity. When the mass fraction is increased the lower values of the relative viscosity along the curvature of the results are limited by the liquidus line and at the top, the boiling point of the solution. The mass fraction of one can be considered as an asymptote since the urea is then in a solid state and thus has no viscosity. This assumption holds until the temperature reaches the urea melting temperature of $405.85 \mathrm{~K}$ [23].

From Fig. 7 it shows that variation in temperature causes some deviation from the mean curvature of the results. When the temperature decreases the results tend to curve downward, see Fig. 8. In lower urea mass fractions, approximately below 0.5 , the viscosity reduction due to the temperature change is slower for the UWS than for water. When the urea mass fraction increases over 0.6 , this behavior attenuates.

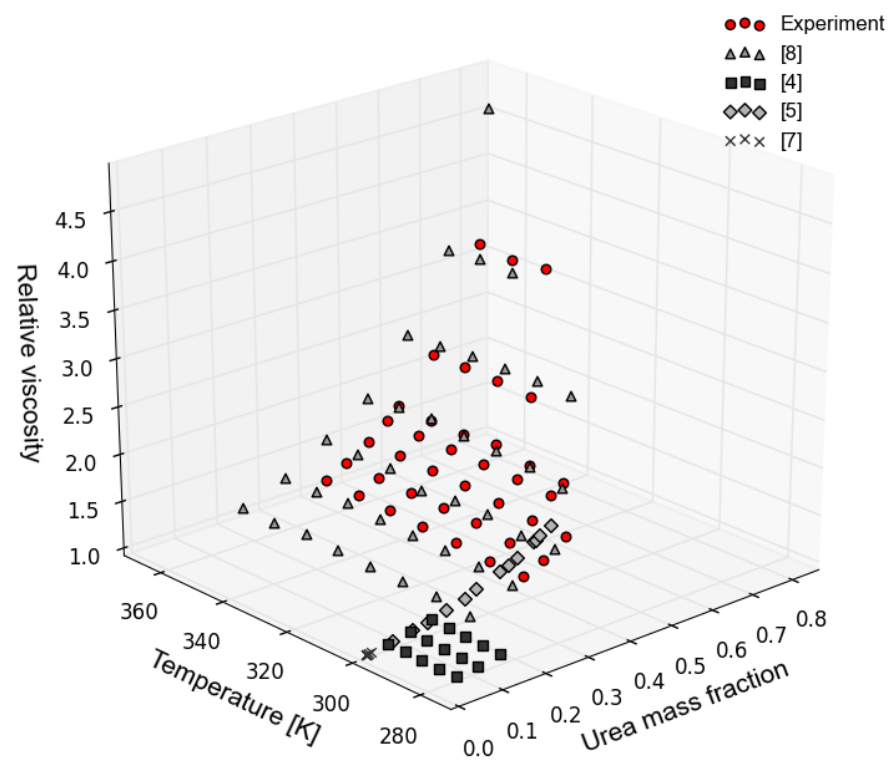

Fig. 8 A 3D-scatter plot of the data from the literature $[4,5,7,8]$ and from our measurements

In an oversaturated solution urea starts to precipitate into the solution. The liquid part of the two-phase solution has the viscosity of fully saturated solution whereas the whole solution becomes slurry-like. According to Yeoh, when the solid concentration in the solution remains below $4 \%$ by weight, the fluid viscous behavior is Newtonian but above this, the solution adopts a pseudo-plastic behavior [24].

In order to obtain the relation of the temperature and urea mass fraction to the relative viscosity, a surface fit was defined from all the data points using the software OriginPro 2016. A Lorentz2D function from the Origin 2016 library, Equation 6, was used for the surface fitting:

$$
v_{r e l}=z_{0}+\frac{A}{\left[1+\left(\frac{x-x_{c}}{w_{1}}\right)^{2}\right]\left[1+\left(\frac{y-y_{c}}{w_{2}}\right)^{2}\right]}
$$

where the parameters $\mathrm{z}_{0}, \mathrm{~A}, \mathrm{x}_{\mathrm{c}}, \mathrm{y}_{\mathrm{c}}, \mathrm{w}_{1}$ and, $\mathrm{w}_{2}$ are optimized to match the data. Parameter $\mathrm{x}$ was assigned to the urea mass fraction and $\mathrm{y}$ to the temperature. The Chi-square tolerance criteria for the surface fit was set to $1 \mathrm{E}-9$ and it was reached in 10 iterations. The resulting surface is shown in Fig. 9 and the model parameters are presented in Table 4. Relative viscosity minimum and maximum residuals from the measured data were -0.111 and 0.0813 , respectively. The standard deviation of the residuals was 0.0249 . 


\begin{tabular}{ccccccc}
\hline Parameter & $\mathbf{z}_{\mathbf{0}}$ & $\mathbf{A}$ & $\mathbf{x}_{\mathbf{c}}$ & $\mathbf{w}_{\mathbf{1}}$ & $\mathbf{y}_{\mathbf{c}}$ & $\mathbf{w}_{\mathbf{2}}$ \\
Value & 0.61544 & 6.7633 & 1.00611 & 0.25743 & 340.6064 & 141.8424 \\
Standard error & 0.01608 & 0.52097 & 0.01596 & 0.00431 & 1.64086 & 8.05579 \\
\hline
\end{tabular}

Table 4 Parameter values and their standard error in the surface fit function

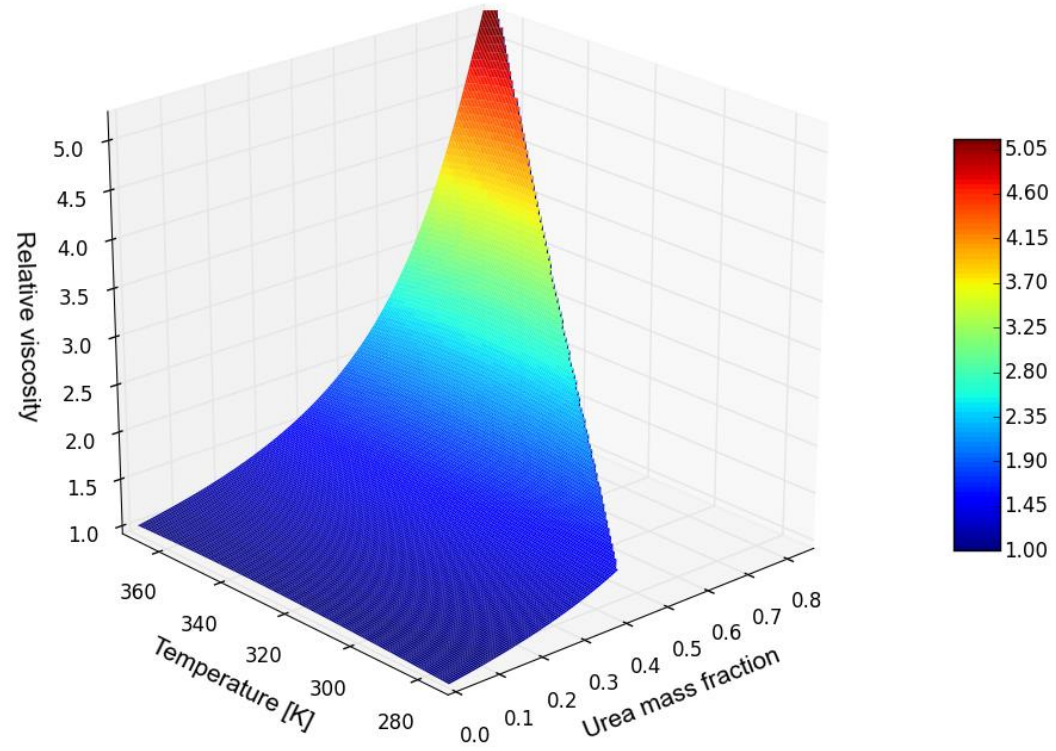

Fig. 9 A surface fit of the measured and literature data where the surface function is expressed by Equation 6 with the parameters presented in Table 4.

The Lorentz2D function describes a pike where the offset is $\mathrm{z}_{0}$, the height is $\mathrm{A}, \mathrm{w}_{1}$ is the width in the $\mathrm{x}$-direction (mass fraction), $w_{2}$ is the width in the $y$-direction (temperature), and $x_{c}$ and $y_{c}$ are peak center locations, respectively. Since the $\mathrm{y}$-axis is assigned to the temperature, the peak which the function describes, is wide when compared to the peak's $\mathrm{x}$ width. The defined area, where the measurements are made, is a narrow slice of the complete peak function. For the surface fit this presents a problem when the value for the $\mathrm{w}_{2}$ parameter is iterated. Similarly, the parameter A, has a large error in relation to its' value. The number of measurement points decreases towards the peak of the function, giving a larger error to parameter A.

\subsection{Surface tension}

The only surface tension values acquirable from the literature were from the chemical company BASF [9] and Birkhold et al. [11], which are $65 \mathrm{mN} / \mathrm{m}$ at ambient temperature of $293.15 \mathrm{~K}$ for a 0.325 urea mass fraction and $75 \mathrm{mN} / \mathrm{m}$ at $303.15 \mathrm{~K}$, respectively. In our measurements the corresponding value for the surface tension was $74.4 \mathrm{mN} / \mathrm{m}$ at 293.15 $\mathrm{K}$.

A linear correlation was calculated from the measured surface tension values with Origin Pro 2016 software, see Fig.

10. The equation for the linear correlation is:

$$
\sigma=10.704 \cdot \gamma+70.825
$$

where $\gamma$ is the urea mass fraction. The $\mathrm{R}^{2}$-value for the linear fit in 0.9385 . The standard error for the intercept and slope of the fit are presented in Table 5.

\begin{tabular}{ccc}
\hline & Intercept & Slope \\
Standard error & 0.44634 & 1.04826 \\
\hline
\end{tabular}


Table 5. Standard error of the intercept and slope of the linear fit

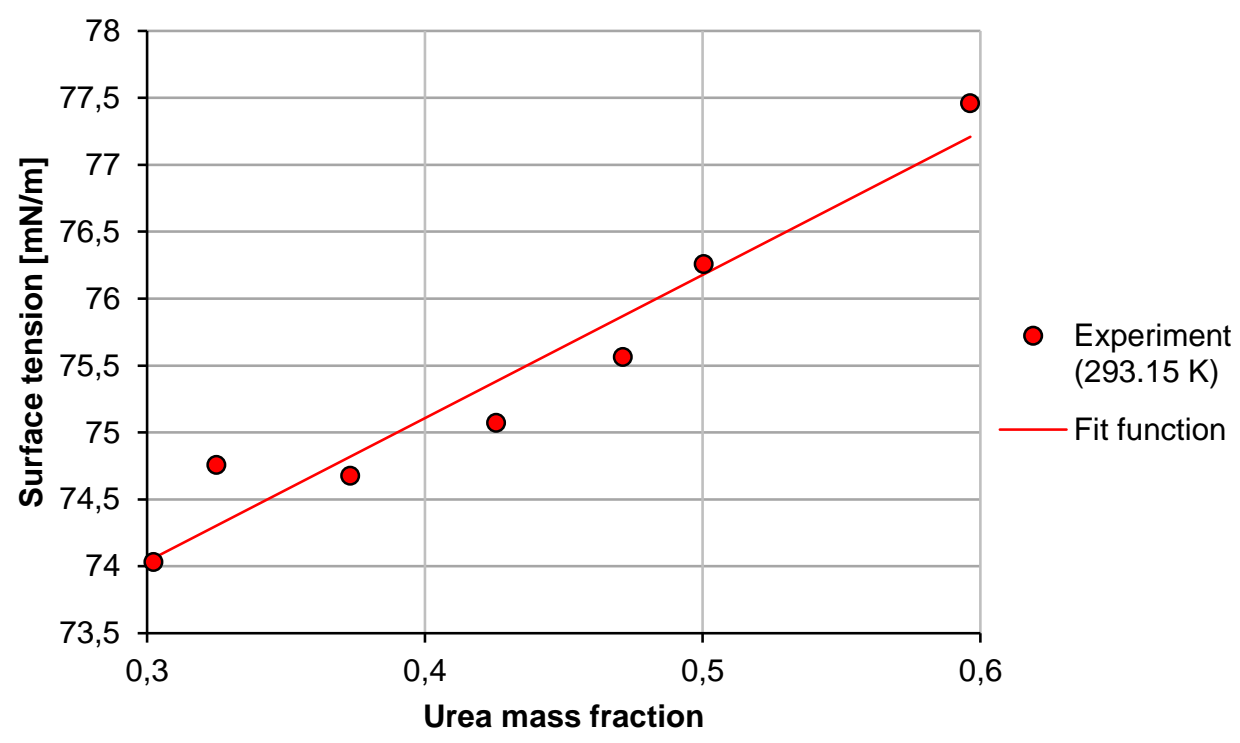

Fig. 10 A linear fit calculated from the surface tension measurements. The solid line represents the fit function presented in Equation 7

As Fig. 10. shows, the surface tension of the solution increases as the amount of urea increases. This is believed to be due to increasing number of hydrogen bonds between the water and urea molecules in the solution. The role of urea in a water solution has been widely studied and there are still uncertainties concerning the structure of a urea-water solution. A good summary of the made studies is presented in Bandyopadhyay et al. [25] where urea-water interactions are studied on a molecular scale. 


\section{Conclusion}

In this article the kinematic viscosity of a UWS was measured in a temperature range from 293.15 to $353.15 \mathrm{~K}$ in $10 \mathrm{~K}$ increments for urea mass fractions from 0.325 to 0.7 and the surface tension of the UWS was measured at room temperature with varying mass fractions from 0.325 to 0.596 . For a comparison with the literature data, the measured kinematic viscosity was changed to relative viscosity. The density values required for the conversation were obtained from the literature and a specific gravity for the UWS was calculated. An exponential function was fit to describe the concentration and temperature effect on the density of the UWS. Lastly, the measured values were compared with the values from the literature and a surface was fit to describe the concentration and temperature dependence on the viscosity of the UWS in the under-saturated region.

The measurements made in this study were found to be in line with the literature data. Slight deviations were observed between the results with Jäger et al. [8] in urea mass fractions over 0.5 . The surface fit presented describes the UWS relative viscosity concentration and temperature dependency reasonably well. The absolute value of maximum difference for the relative viscosity between the data points and the fitted surface was 0.111 and the average was 0.0249 .

In SCR applications the mass fraction of injected UWS is usually 0.325. For CFD simulations the presented viscosity, density, and surface tension models only describe a part of the droplets' evolution in the exhaust system. Wang et al. [26] observed nonhomogeneous droplet evaporation in their experiments. According to their study droplet evaporation can be divided into several groups according to ambient temperature. As the temperature increases the droplet evaporation behavior changes from linear to two-stage evaporation and at temperatures above $573 \mathrm{~K}$ micro explosions start occurring. The viscosity model presented in this study only takes into account liquid droplets without precipitated urea.

Further research needs to be done on the solution properties since they are not defined for the complete range of the droplets' evolution in the system. This should be done parallel with evaporation models to predict the urea precipitating in the droplet and its' effect on the droplet properties. Once the material properties of the droplet are known, dropletwall-interaction models can be better optimized to represent the UWS leading to better fluid film and deposit risk evaluations. 


\section{References}

1. Koebel, M., Elsener, M. \& Kleemann, M. 2000, "Urea-SCR: a promising technique to reduce NOx emissions from automotive diesel engines", Catalysis Today, vol. 59, no. 3, pp. 335-345.

2. Yarin, A.L. 2006, Drop impact dynamics: Splashing, spreading, receding, bouncing..

3. Smith, H., Lauer, T., Mayer, M. \& Pierson, S. 2014, "Optical and Numerical Investigations on the Mechanisms of Deposit Formation in SCR Systems", SAE International Journal of Fuels and Lubricants, vol. 7 , no. 2 , pp. 525-542.

4. Chadwell, H. \& Asnes, B. 1930, "THE VISCOSITIES OF SEVERAL AQUEOUS SOLUTIONS OF ORGANIC SUBSTANCES. II”, Journal of the American Chemical Society, vol. 52, no. 9, pp. 3507-3518.

5. Kawahara K. \& Tanford C. 1966, "Viscosity and density of aqueous solutions of urea and guanidine hydrochloride.”, Journal of Biological Chemistry, vol. 241, no. 13, pp. 3228-3232.

6. Toryanik A.I. \& Topalova E.M. 1986, "Effect of urea on the structure of water over a wide temperature range”, Journal of Structural Chemistry, vol. 26, no. 5, pp. 830-832.

7. Jones, G. \& Talley, S.K. 1933, "The viscosity of aqueous solutions as a function of the concentration", Journal of the American Chemical Society, vol. 55, no. 2, pp. 624-642.

8. Jäger, J., Nývlt, J., Horaček, S. \& Gottfried, J. 1965, "Viskositäten von harnstofflösungen”, Collection of Czechoslovak Chemical Communications, vol. 30, no. 6, pp. 2117-2121.

9. BASF, AdBlue Technical Leaflet, 2006, (http://www.gabriels.be/sites/gabriels/files/pdf/technische_fiche_adblue-_engels.pdf) 30.12.2015

10. Perman, E.P. \& Lovett, T. 1926, "Vapour pressure and heat of dilution of aqueous solutions", Transactions of the Faraday Society, vol. 22, pp. 1-19.

11. Birkhold, F., Meingast, U., Wassermann, P. \& Deutschmann, O. 2006, “Analysis of the injection of ureawater-solution for automotive SCR DeNOx-systems: Modeling of two-phase flow and spray/wallinteraction", SAE Technical Papers, .

12. Ström, H., Lundström, A. \& Andersson, B. 2009, "Choice of urea-spray models in CFD simulations of ureaSCR systems”, Chemical Engineering Journal, vol. 150, no. 1, pp. 69-82.

13. LAUDA - Operating instructions, iVisc, http://www.lauda.de/fileadmin/user_upload/lauda/Documents/Betriebsanleitungen/Englisch/YAME0023_iVi sc_09-09-28_fs.pdf, 10.12.2015 (PDF)

14. Cannon M.R., Manning R.E. \& Bell J.D. 1960, "Viscosity measurement: The kinetic energy correction and a new viscometer", Analytical Chemistry, vol. 32, no. 3, pp. 355-358.

15. Babkina T.S. \& Kuznetsov A.V. 2010, "Phase equilibria in binary subsystems of urea-biuret-water system", Journal of Thermal Analysis and Calorimetry, vol. 101, no. 1, pp. 33-40.

16. Speyers, C. 1902, "Solubilities of some carbon compounds and densities of their solutions", American Journal of Science, , no. 82, pp. 293-302.

17. Pinck L.A. \& Kelly M.A. 1925, “The solubility of urea in water”, Journal of the American Chemical Society, vol. 47, no. 8, pp. 2170-2172. 
18. Kakinuma H. 1941, "The solubility of urea in water", Journal of Physical Chemistry, vol. 45, no. 6, pp. 10451046.

19. Miller Jr. F.W. \& Dittmar H.R. 1934, "The solubility of urea in water. The heat of fusion of urea", Journal of the American Chemical Society, vol. 56, no. 4, pp. 848-849.

20. The International Association for the Properties of Water and Steam, 2014, Revised Release on Surface Tension of Ordinary Water Substance

21. Kell G.S. 1975, "Density, thermal expansivity, and compressibility of liquid water from $0^{\circ}$ to $150^{\circ} \mathrm{C}$ : Correlations and tables for atmospheric pressure and saturation reviewed and expressed on 1968 temperature scale", Journal of Chemical and Engineering Data, vol. 20, no. 1, pp. 97-105.

22. Release on the IAPWS Formulation 2008 for the Viscosity of Ordinary Water Substance. Berlin, Germany September 2008, available at http://www.iapws.org

23. Hawley, G.G. \& Lewis, R.J. 1997, Hawley’s condensed chemical dictionary, Van Nostrand Reinhold.

24. Yeoh, G.H. \& Tu, J. 2010, "Chapter 5 - Liquid-Particle Flows" in Computational Techniques for Multiphase Flows, eds. G.H. Yeoh \& J. Tu, Butterworth-Heinemann, Oxford, pp. 313-349.

25. Bandyopadhyay, D., Mohan, S., Ghosh, S.K. \& Choudhury, N. 2014, "Molecular dynamics simulation of aqueous urea solution: Is urea a structure breaker?”, Journal of Physical Chemistry B, vol. 118, no. 40, pp. 11757-11768.

26. Wang, T.J., Baek, S.W., Lee, S.Y., Kang, D.H. \& Yeo, G.K. 2009, "Experimental investigation on evaporation of urea-water-solution droplet for SCR applications”, AIChE Journal, vol. 55, no. 12, pp. 3267 3276. 